\title{
Transmission Coefficient of an Electron through a Heterostructure with Nanometer-Thick Trapezoidal Barrier Grown on an Anisotropic Material
}

\author{
Lilik Hasanah ${ }^{1}$, Fatimah A. Noor, Khairurrijal, Mikrajuddin, Toto Winata \& \\ Sukirno \\ Faculty of Mathematics and Natural Sciences, Institut Teknologi Bandung, \\ Jalan Ganesha 10, Bandung 40132
}

\begin{abstract}
Transmission coefficient of an electron incident on a heterostructure potential with nanometer-thick trapezoidal barrier grown on anisotropic materials are derived by solving the effective-mass equation including offdiagonal effective-mass tensor elements. The boundary condition for an electron wave function (under the effective-mass approximation) at a heterostructure anisotropic junction is suggested and included in the calculation. The analytic expressions are applied to the $\mathrm{Si}(110) / \mathrm{Si}_{0.5} \mathrm{Ge}_{0.5} / \mathrm{Si}(110)$ heterostructure, in which the $\mathrm{SiGe}$ barrier thickness is several nanometers. It is assumed that the direction of propagation of the electrons makes an arbitrary angle with respect to the interfaces of the heterostructure and the effective mass of the electron is position dependent. The transmission coefficient is calculated for energy below the barrier height, varying the applied voltage to the barrier. The transmission coefficient depends on the valley where the electron belongs and it is not symmetric with respect to the incidence angle.
\end{abstract}

Keywords: Anisotropic material; heterostructure; nanometer-thick barrier; transmission coefficient; tunneling time.

\section{$1 \quad$ Introduction}

Since last half century, the tunneling phenomenon through a potential barrier is still of interest in the study of quantum transport in heterostructures. Paranjape studied transmission coefficient of an electron in an isotropic heterostructure with different effective masses [1]. Kim and Lee derived the transmission coefficient of an electron tunneling through a barrier of an anisotropic heterostructure by solving the effective-mass equation including off-diagonal effective-mass tensor elements [2],[3]. The effects of different effective masses to the heterostructure were also included but they did not consider the effects of voltage applied to the barrier in which the square barrier becomes trapezoidal one. In this paper, we report the derivation and the calculation of the transmission coefficient of an electron through a heterostructure with a

${ }^{1}$ On leave from Jurusan Fisika, FPMIPA, Universitas Pendidikan Indonesia, Bandung.

Makalah diterima redaksi tanggal 15 Desember 2005, revisi diterima tanggal 29 Maret 2006. 
nanometer-thick trapezoidal barrier grown on an anisotropic material, including the effect of applied voltage to the barrier.

\section{$2 \quad$ Theoretical Model}

The conduction band energy diagram of a heterostructure is shown in Fig 1 with the potential profile is expressed as:

$$
V(z)=\left\{\begin{array}{ccc}
0 & \text { for } & z \leq 0 \\
\Phi-\frac{e V_{b}}{d} z & \text { for } & 0<z<d \\
-e V_{b} & \text { for } & z \geq d .
\end{array}\right.
$$

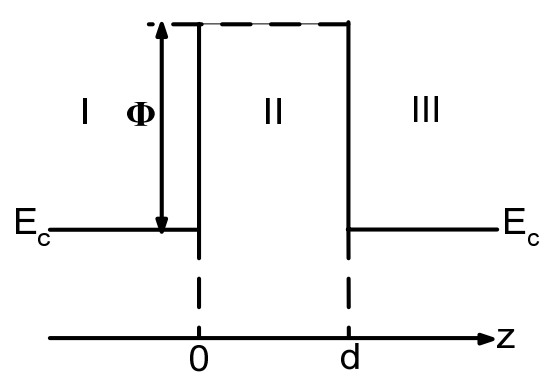

(a)

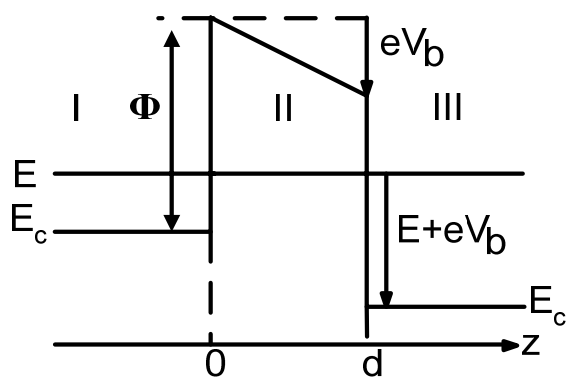

(b)

Figure 1 The potential profile of a heterostructure without a bias voltage (a) and with the application of a voltage to the barrier (b).

Here, the barrier width and height are $d$ and $\Phi$, respectively. The voltage applied to the barrier is $V_{b}$ with $e$ is the electronic charge. The electron is incident from region I to the potential barrier (region II), in which the material of the region I is the same as that of the region III.

The Hamiltonian for general anisotropic materials is [2]

$$
H=\frac{1}{2 m_{o}} \mathbf{p}^{T} \alpha(\mathbf{r}) \mathbf{p}+V(\mathbf{r}),
$$

where $m_{o}$ is the free electron mass, $\mathbf{p}$ is the momentum vector, $\left(1 / m_{o}\right) \alpha(\mathbf{r})$ is the inverse effective-mass tensor and $V(\mathbf{r})$ is the potential energy. The effective mass of the electron and potential are dependent only on the $\mathrm{z}$ direction. The wave function of the effective-mass equation with the Hamiltonian in Eq. (2) is given as [2]:

$$
\psi(\mathbf{r})=\varphi(z) \exp (-i \gamma z) \exp \left(i\left(k_{x} x+k_{y} y\right)\right)
$$




$$
\text { and } \gamma=\frac{k_{x} \alpha_{x z}+k_{y} \alpha_{y z}}{\alpha_{z z}}
$$

is wave number parallel to the interface.

By employing the separation variable to Eq. (2), it is easily found that $\varphi(z)$ satisfies the one dimensional Schrödinger-like equation:

$$
-\frac{\hbar^{2}}{2 m_{o}} \alpha_{z z, l} \frac{\partial^{2} \varphi(z)}{\partial z^{2}}+V(z) \varphi(z)=E_{z} \varphi(z),
$$

where $\hbar$ is the reduced Planck constant, the subscript $l$ in $\alpha_{z z, l}$ denotes each region in Fig. 1 and

$$
E_{z}=E-\frac{\hbar^{2}}{2 m_{o}} \sum_{i, j \in\{x, y\}} \beta_{i j} k_{i} k_{j} .
$$

Here,

$$
E=\sum_{i, j \in\{x, y, z\}} \frac{\hbar^{2}}{2 m_{o}} \alpha_{i j, 1} k_{i} k_{j}
$$

is the total energy,

$$
\beta_{i j}=\alpha_{i j}-\frac{\alpha_{i z} \alpha_{z j}}{\alpha_{z z}},
$$

and $\alpha_{i j}$ is the effective mass tensor element.

The time-independent electron wave function in each region is therefore written as

$$
\begin{aligned}
& \psi_{1}(\vec{r})=\left(A e^{i k_{1} z}+B e^{-i k_{1} z}\right) e^{-\left(i \gamma_{1} z\right)} e^{-\left(i k_{x} x+i k_{y} y\right)}, \text { for } z \leq 0, \\
& \psi_{2}(\vec{r})=\left(C e^{-\int_{0}^{z} k_{2}(z) d z}+D e^{\int_{0}^{z} k_{2}(z) d z}\right) e^{-\left(i \gamma_{2} z\right)} e^{-\left(i k_{x} x+i k_{y} y\right)}, \text { for } 0<z<d, \\
& \psi_{3}(\vec{r})=F e^{i k_{3} z} e^{-\left(i \gamma_{1} z\right)} e^{-\left(i k_{x} x+i k_{y} y\right)}, \text { for } z \geq d
\end{aligned}
$$

The incident wave $A \exp \left(i k_{1} z\right)$ has the wave number $k_{1}$ which is given as

$$
k_{1}=\left\{\frac{2 m_{o} E_{z}}{\hbar^{2}} \frac{1}{\alpha_{z z, 1}}\right\}^{1 / 2},
$$


where $E_{z}$ is smaller than the barrier height $\Phi$. The wave numbers $k_{2}(z)$ and $k_{3}$ are expressed, respectively, as follows

$$
k_{2}(z)=\left\{\frac{2 m_{0}}{\hbar^{2}} \frac{1}{\alpha_{z z, 2}}\left(\Phi-e \frac{V_{b}}{d} z\right)-\frac{\alpha_{z z, 1}}{\alpha_{z z, 2}} k_{1}^{2}-\frac{1}{\alpha_{z z, 2}} \sum_{i, j \in(x, y)}\left(\beta_{i j, 1}-\beta_{i j, 2}\right) k_{i} k_{j}\right\}^{1 / 2},
$$

and

$$
k_{3}=\left\{\frac{2 m_{o}\left(E_{z}+e V_{b}\right)}{\hbar^{2}} \frac{1}{\alpha_{z z, 1}}\right\}^{1 / 2} .
$$

By applying the boundary conditions at $z=0$ dan $z=\mathrm{d}$, which are written as follows [3]:

$$
\begin{aligned}
& \psi_{I}\left(z=0^{-}\right)=\psi_{2}\left(z=0^{+}\right), \\
& \frac{1}{m_{o}}\left[\alpha_{z x, I} \frac{d \psi_{1}}{d z}+\alpha_{z y, I} \frac{d \psi_{1}}{d z}+\alpha_{z z, I} \frac{d \psi_{1}}{d z}\right]_{z=0^{-}} \\
& =\frac{1}{m_{o}}\left[\alpha_{z x, 2} \frac{d \psi_{2}}{d z}+\alpha_{z y, 2} \frac{d \psi_{2}}{d z}+\alpha_{z z, 2} \frac{d \psi_{2}}{d z}\right]_{z=0^{+}}, \\
& \psi_{2}\left(z=d^{-}\right)=\psi_{3}\left(z=d^{+}\right), \\
& \frac{1}{m_{o}}\left[\alpha_{z x, 2} \frac{d \psi_{2}}{d z}+\alpha_{z y, 2} \frac{d \psi_{2}}{d z}+\alpha_{z z, 2} \frac{d \psi_{2}}{d z}\right]_{z=d^{-}} \\
& =\frac{1}{m_{o}}\left[\alpha_{z x, 1} \frac{d \psi_{3}}{d z}+\alpha_{z y, 1} \frac{d \psi_{3}}{d z}+\alpha_{z z, 1} \frac{d \psi_{3}}{d z}\right]_{z=d^{+}},
\end{aligned}
$$

we obtain the transmission amplitude $T_{a}$ which is defined as

$$
T_{a}=\frac{F}{A}=G \exp (i \phi) .
$$

Here,

$$
G=\frac{2 k_{1} k_{2}^{d}}{\left(P^{2} \operatorname{Sinh}^{2}(u)+Q^{2} \operatorname{Cosh}^{2}(u)\right)^{1 / 2}}
$$

is the magnitude and

$$
\phi=\left[\tan ^{-1}\left(\frac{P}{Q}\right) \tanh (u)\right]-k_{3} d+\left(\gamma_{1}-\gamma_{2}\right) d
$$


is the phase of $T_{a}$,

$$
\begin{aligned}
& P=\left(\frac{\alpha_{z z, 1}}{\alpha_{z z, 2}} k_{1} k_{3}-\frac{\alpha_{z z, 2}}{\alpha_{z z, 1}} k_{2}^{0} k_{2}^{d}\right), \\
& Q=\left(k_{3} k_{2}^{0}+k_{1} k_{2}^{d}\right), \\
& k_{2}^{0}=k_{2}(z=0), \\
& k_{2}^{d}=k_{2}(z=d),
\end{aligned}
$$

and

$$
u=\int_{0}^{d} k_{2}(z) d z
$$

The transmission coefficient is easily obtained from Eq. (16) by employing the expression

$$
T=T_{a}^{*} T_{a} .
$$

If the voltage applied to the barrier is zero, then $k_{2}^{0}=k_{2}^{d}=k_{2}, k_{1}=k_{3}$, and the expressions in Eqs. (17) and (18) will be the same as that given by Lee [2], in which

$$
\begin{aligned}
& G=\frac{2 k_{1} k_{2}}{\left(P^{2} \operatorname{Sinh}^{2}(u)+Q^{2} \operatorname{Cosh}^{2}(u)\right)^{1 / 2},} \\
& \phi=\left[\tan ^{-1}\left(\frac{P}{Q}\right) \tanh (u)\right]-k_{3} d+\left(\gamma_{1}-\gamma_{2}\right) d,
\end{aligned}
$$

where

$$
\begin{aligned}
& P=\left(\frac{\alpha_{z z, I}}{\alpha_{z z, 2}} k_{1}^{2}-\frac{\alpha_{z z, 2}}{\alpha_{z z, I}} k_{2}^{2}\right), \\
& Q=2 k_{1} k_{2},
\end{aligned}
$$

and

$$
u=k_{2} d \text {. }
$$




\section{$3 \quad$ Results and Discussion}

The model used in the numerical calculation is shown in Fig. 1 with a potential barrier is a strained $\mathrm{Si}_{0.5} \mathrm{Ge}_{0.5}$ potential barrier grown on $\mathrm{Si}$ (110). The width of the barrier $\mathrm{d}$ is $50 \stackrel{\circ}{\mathrm{A}}$ and the band discontinuity $\Phi$ is taken as $216 \mathrm{meV}$ [2].

There are four equivalent valleys in the conduction bands of $\mathrm{Si}(110)$ and strained $\mathrm{Si}_{0.5} \mathrm{Ge}_{0.5}$. The effective mass tensor elements of these four valleys are not the same. There are two groups of valleys in $\mathrm{Si}(110)$ and $\mathrm{Si}_{0.5} \mathrm{Ge}_{0.5}$. The inverse effective inverse tensor used in Eq. (2) are related to the tensor elements $\alpha_{i j}$ shown in Table 1 [2]. In Table 1, we see that one group (valley 1) has positive $\alpha_{y z}$, while another one (valley 2) has negative $\alpha_{y z}[3]$. We denote the group that has positive $\alpha_{y z}$ as valley 1 and the other as valley 2 . Therefore, the calculated results dependent on the group which electron belongs.

Table 1 Tensor elements $\left(\alpha_{i j}\right)$ used in the numerical calculation.

\begin{tabular}{|c|ccc|ccc|}
\hline Valley & \multicolumn{2}{|c|}{ Region I dan III (Si $\mathbf{[ 1 1 0 ]})$} & \multicolumn{3}{|c|}{ Region } & $\mathbf{I I}\left(\mathbf{S i}_{\mathbf{0}, \mathbf{5}} \mathbf{G e}_{\mathbf{0}, \mathbf{5}}\right)$ \\
\hline 1 & 5.26 & 0 & 0 & 6.45 & 0 & 0 \\
& 0 & 3.14 & 2.12 & 0 & 4.56 & 2.74 \\
& 0 & 2.12 & 3.14 & 0 & 2.74 & 4.56 \\
\hline 2 & 5.26 & 0 & 0 & 6.45 & 0 & 0 \\
& 0 & 3.14 & -2.12 & 0 & 4.56 & -2.74 \\
& 0 & -2.12 & 3.14 & 0 & -2.74 & 4.56 \\
\hline
\end{tabular}

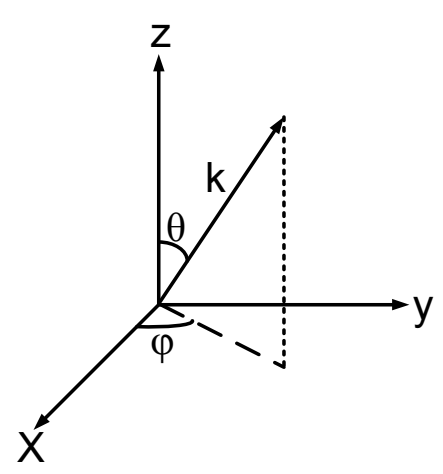

Figure 2 The coordinate system used in the analysis.

Figure 2 shows the chosen coordinate system. We take the position where the electron hits the barrier as the origin of the coordinate system. In the spherical coordinate system, Eq. (7) becomes 


$$
\begin{aligned}
E & =\frac{\hbar^{2}}{2 m_{o}}\left\{\alpha_{x x 1} k^{2} \sin ^{2} \theta \cos ^{2} \varphi+\alpha_{y y 1} k^{2} \sin ^{2} \theta \sin ^{2} \varphi+\alpha_{z z 1} k^{2} \cos ^{2} \theta\right. \\
& +2\left(\alpha_{x y 1} k^{2} \sin ^{2} \theta \cos \varphi \sin \varphi+\alpha_{y z 1} k^{2} \sin ^{2} \theta \cos \theta \sin \varphi\right. \\
& \left.\left.+\alpha_{z x 1} k^{2} \sin ^{2} \theta \cos \theta \cos \varphi\right)\right\}
\end{aligned}
$$

We calculated the transmission coefficient for the angle of incidence for $\mathbf{k}$ (the wave vector of incident electron) varying from $-90^{\circ}$ to $90^{\circ}$ with incident energies of $25 \mathrm{meV}, 75 \mathrm{meV}$ and $150 \mathrm{meV}$ and varying the applied voltage from $50 \mathrm{mV}$ to $150 \mathrm{mV}$. The incident angles are $\theta$ and $\varphi$, but we fix $\varphi$ to $\pi / 2$ for simplicity and change only $\theta$.

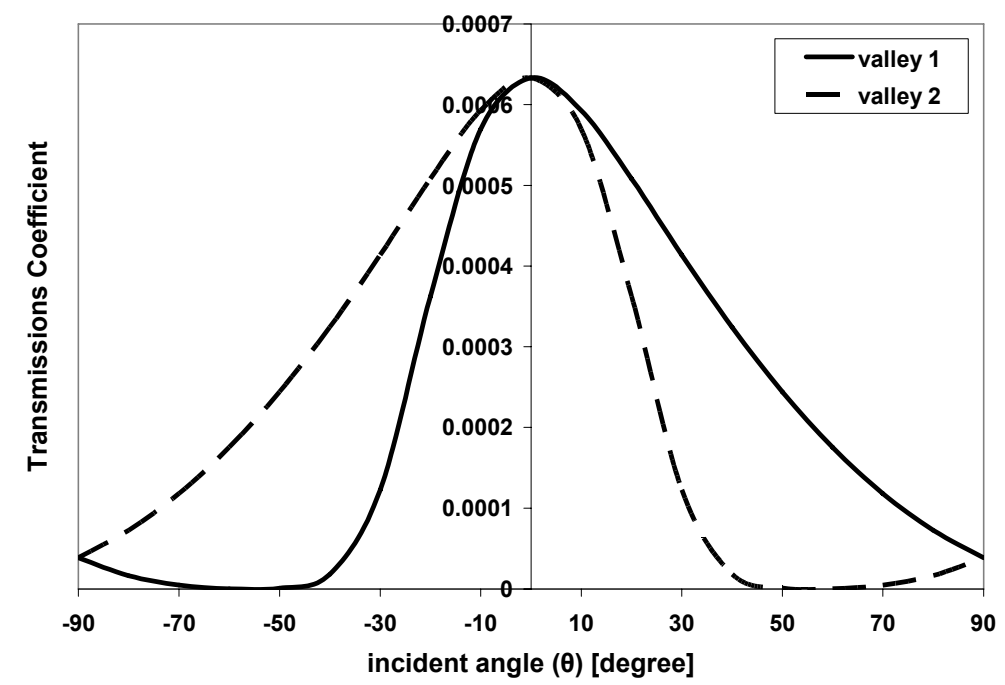

Figure 3 The transmission coefficient for the incident angle varying from $-90^{\circ}$ to $90^{\circ}$ with incident energy of $75 \mathrm{meV}$ and applied voltage of $50 \mathrm{mV}$.

The transmission coefficient as a function of incident angle for incident energy of $75 \mathrm{meV}$ and applied voltage of $50 \mathrm{mV}$ is shown in Fig. 3. The solid line is for electrons in the valley 1 and dashed line is for those in the valley 2 . We can see that the transmission coefficient for electrons in the valley 1 and valley 2 occurs at normal incident. In addition, the transmission coefficient becomes the lowest for $-65^{\circ}<\theta<-45^{\circ}$ (the valley 1) and $45^{\circ}<\theta<65^{\circ}$ (the valley 2). The sign \pm corresponds to valley 1 and 2, respectively. This difference in direction also indicates the anisotropy of the material. It is due to the fact that the motion in the $\mathrm{x}$ and $\mathrm{y}$ directions is not independent of that in the $\mathrm{z}$ direction, but they are mutually coupled by the off-diagonal effective-mass tensors elements[2]. 


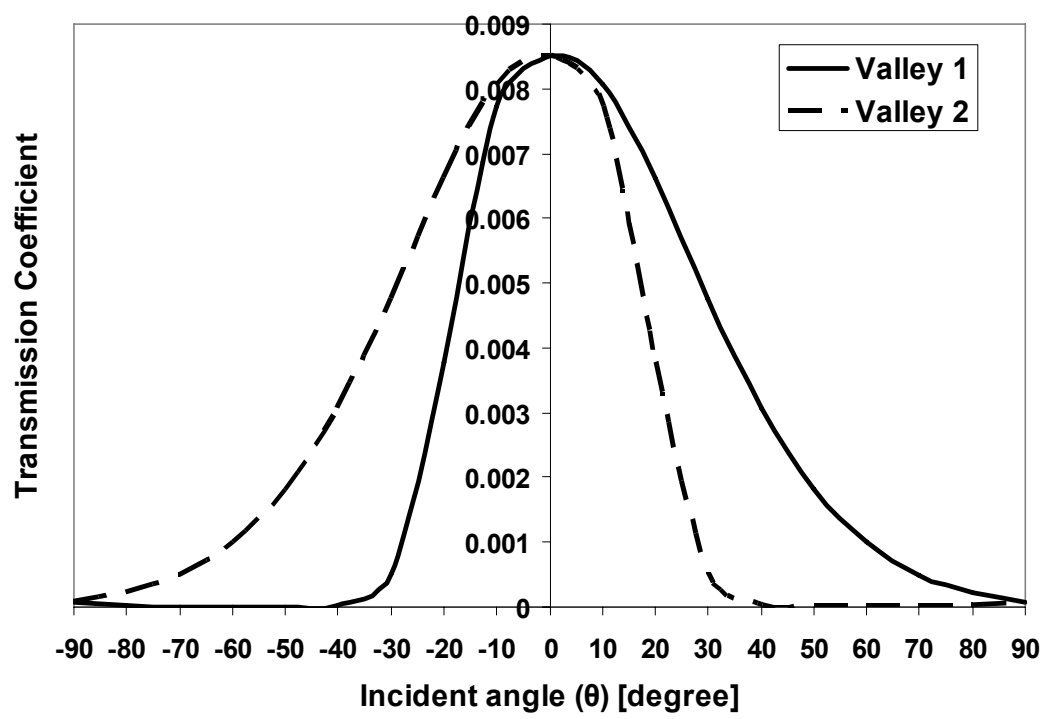

Figure 4 The transmission coefficient for the incident angle varying from $-90^{\circ}$ to $90^{\circ}$ with incident energy of $150 \mathrm{meV}$ and applied voltage of $50 \mathrm{mV}$.

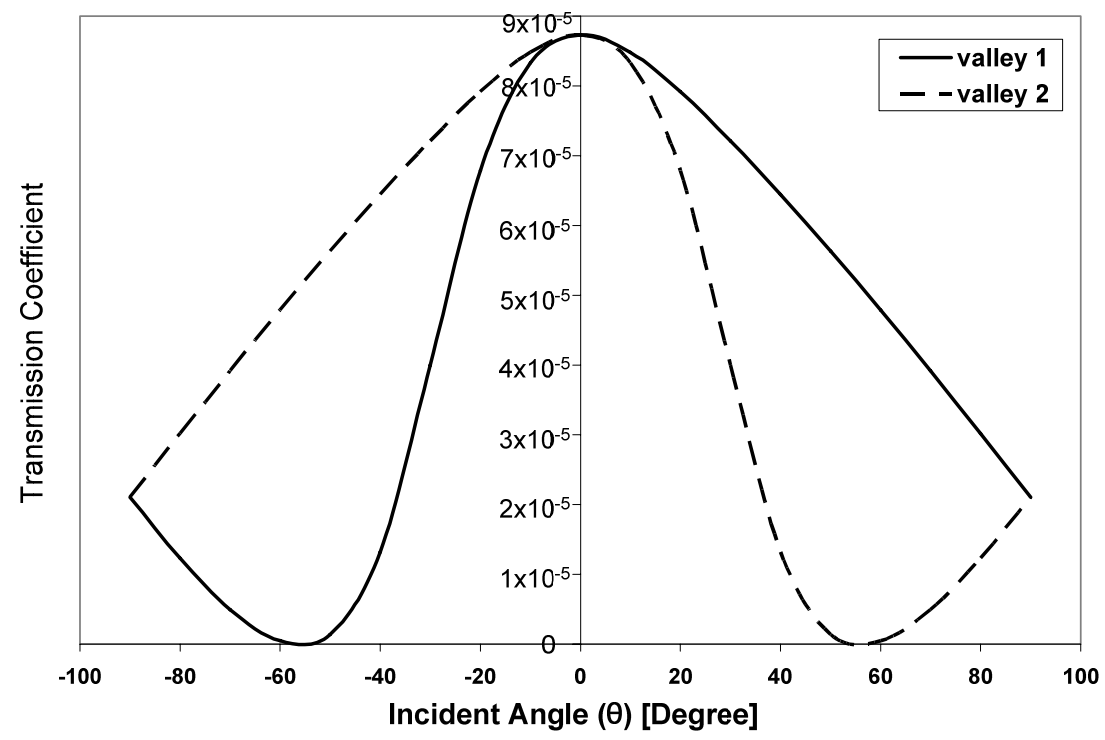

Figure 5 The transmission coefficient for the incident angle varying from $-90^{\circ}$ to $90^{\circ}$ with incident energy of $25 \mathrm{meV}$ and applied voltage of $100 \mathrm{mV}$. 


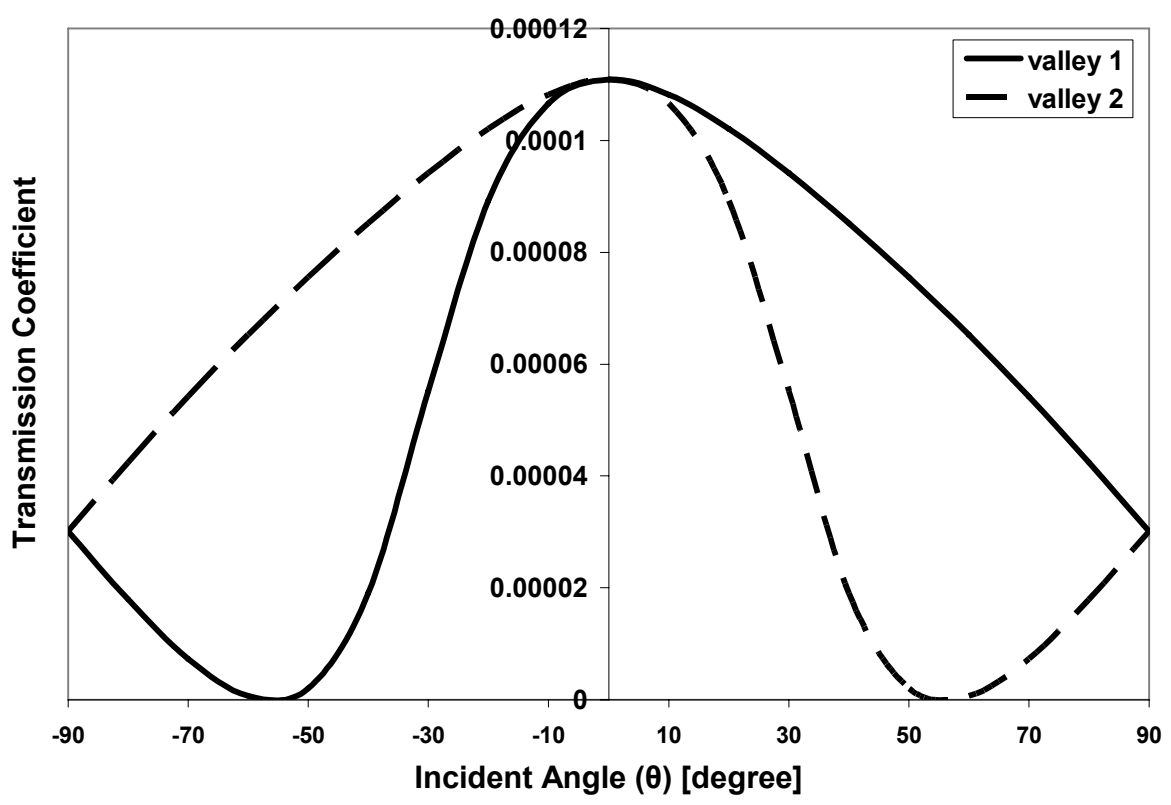

Figure 6 The transmission coefficient for the incident angle varying from $-90^{\circ}$ to $90^{\circ}$ with incident energy of $25 \mathrm{meV}$ and applied voltage of $150 \mathrm{mV}$.

In Fig. 4 the transmission coefficient for incident energy of $150 \mathrm{meV}$ and applied voltage of $50 \mathrm{mV}$ is presented. It is found that electron in the valley 1 and valley 2 have the highest transmission coefficient at about normal incidence. It is noted that the maximum value of the transmission coefficient for the incident energy of $75 \mathrm{meV}$ is lower than that for the incident energy of 150 $\mathrm{meV}$ because the electrons have lower energy so that the probability of electrons to tunnel the barrier is also smaller. The incident angles having the lowest transmission coefficient becomes wider $\left(-80^{\circ}<\theta<-40^{\circ}\right.$ and $\left.40^{\circ}<\theta<80^{\circ}\right)$ as the electron energy increases. It is probably due to the fact that if we increase the electron's incident energy then the energy in z direction is decreases. We also observe that for all valleys, the transmission coefficients are not symmetric with the incidence angle. If we decrease the incident energy, the electrons have lower energy to tunnel the potential barrier so that the probability of tunneling the barrier is smaller than that for the electrons with higher incident energy although bias voltage is increased as shown in Fig. 5. Transmission coefficient in Fig. 5 decrease two order of magnitude compare to transmission coefficient in Fig. 3. But for the same incident energy, the transmission coefficient will increase when the applied voltage to the barrier increased as shown in Fig. 6. In Fig 5 and 6, the maximum transmission coefficient is $9 \times 10^{-5}$ and $11 \times 10^{-5}$, respectively. For the case in Figs. 5 and 6, the transmission coefficient is maximum at normal incident. We also see that, in all valleys, the transmission 
coefficient is not symmetric with the change of sign of incidence angle $(\theta \rightarrow-\theta)$, which confirms the anisotropic of the materials [2].

\section{Conclusion}

We have derived an analytical expression of transmission coefficient of an electron through a nanometer-thick trapezoidal barrier grown on anisotropic materials under non-normal incidence. We included the effect of different effective masses at heterojunction interfaces. The boundary conditions for electron wave functions (under the effective-mass approximation) at heterostructure anisotropic junctions are suggested and included in the calculation. The transmission coefficient will increase if the incident energy is increased. For the same incident energy, the highest value of the transmission coefficient occurs if the applied voltage to the barrier is high. The result shows that the transmission coefficient depends on the valley and it is not symmetric with the angle of incidence.

\section{Acknowledgement}

One of the authors (L.H) would like to thank the Habibie Center for the domestic doctoral scholarship.

\section{References}

1. V.V. Paranjape: Transmission coefficient and stationary-phase tunneling time of an electron through a heterostructure, Phys.Rev. B. Vol 52. No 15, 1995, $10740-10743$.

2. K.-Y. Kim and B. Lee: Transmission coefficient of an electron through a heterostructure barrier grown on anisotropic materials, Phys.Rev. B. Vol 58. No 11, 1998, 6728-6731.

3. K.-Y. Kim and B. Lee: Tunneling time and the post-tunneling position of an electron through a potential barrier in an anisotropic semiconductor, Superlattice Microstruct. Vol. 24. No 6, 1998, 389-397. 